\title{
Polarity determination in the Drosophila eye: a novel role for Unpaired and JAK/STAT signaling
}

\author{
Martin P. Zeidler, ${ }^{1}$ Norbert Perrimon, ${ }^{1,2}$ and David I. Strutt ${ }^{3,4}$ \\ ${ }^{1}$ Department of Genetics, Harvard Medical School, Boston, Massachusetts 02115 USA; ${ }^{2}$ Howard Hughes Medical Institute, \\ ${ }^{3}$ Developmental Genetics Programme, University of Sheffield, Western Bank, Sheffield S10 2TN, UK
}

The JAK/STAT signaling pathway is required for many processes including cytokine signaling, hematopoiesis, gliagenesis, and Drosophila segmentation. In this report we present evidence demonstrating that the JAK/STAT pathway is also central to the establishment of planar polarity during Drosophila eye development. We show that a localized source of the pathway ligand, Unpaired, is present at the midline of the developing eye, which is capable of activating the JAK/STAT pathway over long distances. A gradient of JAK/STAT activity across the DV axis of the eye regulates ommatidial polarity via an unidentified second signal. Additionally, localized Unpaired influences the position of the equator via repression of mirror.

[Key Words: Rotation; ommatidia; equator; unpaired; hopscotch; stat92E]

Received February 12, 1999; revised version accepted April 13, 1999.

The Janus kinase (JAK)/signal transducer and activator of transcription (STAT) pathway is a signal transduction mechanism originally identified in mammals (Schindler et al. 1992). The pathway consists of a transmembrane receptor molecule with which the JAK tyrosine kinase is constitutively associated. Following activation and dimerization of the receptor by ligand binding (Fig. 1A, 1-2), JAK kinase is activated by phosphorylation and then recruits cytosolic STAT to the complex (Fig. 1A, 3). Recruited STAT molecules are then phosphorylated on an invariant tyrosine residue and translocate to the nucleus (Fig. 1A, 4) in which they bind a consensus DNA motif and activate transcription (Fig. 1A, 5 for review, see Darnell 1997; O'Shea 1997). In mammalian systems, the JAK/STAT pathway has been implicated in the transduction of cytokine signaling (Kaplan et al. 1996; Shimoda et al. 1996) as well as the development of the immune system (Macchi et al. 1995; Durbin et al. 1996), mammary glands (Liu et al. 1997), and glial cells (Bonni et al. 1997).

The JAK/STAT pathway is also present in Drosophila, in which a single JAK homolog encoded by the hopscotch (hop) locus (Binari and Perrimon 1994) and a single STAT homolog, called stat92E, have been identified (Hou et al. 1996; Yan et al. 1996b). Mutations in both Drosophila JAK and STAT were first identified by virtue of their distinctive embryonic phenotypes. When the function of either is removed from the embryo, there is partial loss of expression of the pair-rule genes runt and

${ }^{4}$ Corresponding author.

E-MAIL d.strutt@sheffield.ac.uk; FAX 44-114-222-2788. even-skipped and, consequently, some abdominal segments are lost or fused together (Perrimon and Mahowald 1986; Binari and Perrimon 1994; Hou et al. 1996).

Recently, an in vivo ligand activating the JAK/STAT pathway has also been identified in Drosophila. A report by Harrison et al. (1998) presents evidence that the secreted protein Unpaired (Upd) acts as the pathway ligand during embryonic segmentation. Mutations in Upd give an identical embryonic phenotype to loss of hop or stat92E, Upd is expressed in the domains in which JAK/ STAT activity is required during segmentation, and Upd activates JAK in a tissue-culture assay (Harrison et al. 1998).

In addition to its role in embryonic segmentation, the Drosophila JAK/STAT pathway is also required for other developmental events. For instance, Hop regulates proliferation of imaginal tissue (Perrimon and Mahowald 1986) and the dominant gain-of-function (GOF) hop ${ }^{\text {Tuml }}$ allele causes melanotic tumors and hypertrophy of the larval lymph glands (Hanratty and Dearolf 1993; Harrison et al. 1995; Luo et al. 1995). Ectopic Hop expression has also been associated with cell fate transformations (Harrison et al. 1995) and partial loss-of-function (LOF) STAT alleles have been described that display wing vein phenotypes (Yan et al. 1996a). Also, a number of viable alleles of upd have been identified, including outstretched small eye which is probably a regulatory allele (Harrison et al. 1998) and results in both reduced eye size and a heldout wing phenotype.

In this study we investigate the function of Upd and the JAK/STAT pathway in Drosophila eye development and their roles in polarity decisions. The compound eye 


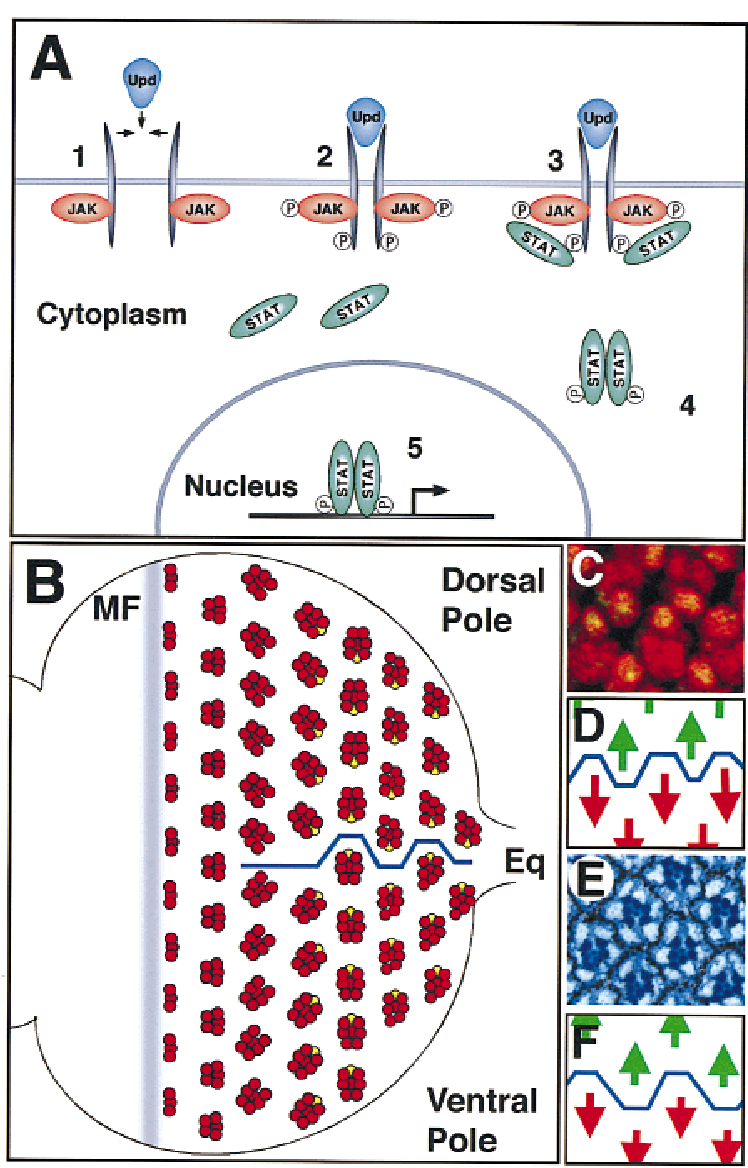

Figure 1. The JAK/STAT pathway and the establishment of dorsoventral polarity in the eye disc. Anterior is to the left and dorsal up, here and in all subsequent figures. $(A)$ The transduction of extracellular signals from the cell membrane to the nucleus by the JAK/STAT signaling pathway. (B) A schematized representation of ommatidial development and rotation. The movement of the morphogenetic furrow (MF) from posterior to anterior across the disc leads to the determination of successive rows of increasingly mature ommatidial clusters (red). Shortly before determination of the R7 photoreceptor (yellow), dorsal and ventral clusters rotate in opposite directions to generate the equator (blue line). (C) Ommatidial clusters at the equator in the third instar eye disc. All photoreceptor nuclei are marked by anti-Elav (red) and the R7 nucleus is also marked by expression of Prospero (green, yellow when overlaid). (D) Schematic representation of $C$ showing the position of the equator (blue line) through this region and the green dorsal arrows and red ventral arrows used to indicate ommatidial polarity. $(E)$ Adult eye section at the equator showing the stereotypical trapezoidal arrangement of rhabdomeres in each adult ommatidial cluster. Each ommatidium is surrounded by a hexagonal array of pigment granules. $(F)$ Schematic representation of $E$ showing position of the equator (blue line) through this region and the green dorsal arrows and red ventral arrows used to indicate ommatidial polarity.

of Drosophila has proved to be a rewarding system for such studies. In this structure, polarity is established at the level of repeating multicellular units (known as ommatidia), which are organized into a precise hexagonal array (for review, see Wolff and Ready 1993). The adult eye is composed of $\sim 800$ ommatidia, each of which forms one facet. Sections through the eye reveal that each ommatidium contains eight photoreceptor cells in a stereotypic trapezoidal arrangement that has two mirror-symmetric forms, a dorsal form present above the dorsoventral (DV) midline, and a ventral form below. An axis of mirror-image symmetry runs along the DV midline and is known as the equator. By analogy to the terrestrial equator, the extreme dorsal and ventral points of the eye are referred to as the poles (Fig. 1B-F).

Differentiation of ommatidia begins during the third instar larval stage when a furrow moves from posterior to anterior over the epithelium of the eye imaginal disc. Each ommatidial unit is born as a bilaterally symmetrical cluster of photoreceptor precursors, which is polarized on its anteroposterior axis (Fig. 1B). The clusters then become polarized on the DV (or equatorial-polar) axis, as the proto-ommatidium rotates via two $45^{\circ}$ steps away from the DV midline, forming the equator (Fig. 1B). It has been suggested that the direction of this rotation is a consequence of a gradient of positional information emanating from either the midline or the polar regions of the disc (e.g., Baker and Rubin 1992; Ma and Moses 1995; Zheng et al. 1995).

A number of recent studies have shed light on some of the mechanisms involved in the positioning of the equator on the DV midline of the eye imaginal disc. It is now clear that a critical step is the activation of Notch activity in a line of cells along the midline, and that this localized activation of Notch is a consequence of the restricted expression of the fringe $(f n g)$ gene product in the ventral half of the disc and the homeodomain transcription factor Mirror (Mirr) in the dorsal half of the disc (McNeill et al. 1997; Cho and Choi 1998; Domínguez and de Celis 1998; Papayannopoulos et al. 1998).

Furthermore, an important role for Wingless (Wg) in polarity determination on the DV axis has been demonstrated. Wg is a secreted protein (and the founder member of the Wnt family of morphogens), which is expressed at the poles of the eye disc. Wg has been shown to act as an activator of mirr expression; increasing the levels of Wg expression in the eye disc shifts mirr expression and the position of the equator ventrally, whereas reduction of $w g$ function shifts mirr expression dorsally (Reifegerste et al. 1997; Heberlein et al. 1998). Additionally, it has been shown convincingly that a gradient of $\mathrm{Wg}$ signaling across the DV axis of the eye disc regulates ommatidial polarity such that the lowest point of Wg signaling coincides with the equator (Wehrli and Tomlinson 1998).

This paper is the first detailed analysis of Upd/JAK/ STAT pathway function during Drosophila eye development. We show that Upd is necessary to determine the position of the equator via regulation of mirr. Furthermore, loss of the intracellular pathway components (JAK/STAT) produces ectopic axes of mirror-image symmetry characterized by nonautonomous inversions of ommatidial polarity, a phenotype also produced by Upd misexpression. We have used a novel in vivo assay for 
JAK/STAT activity on the basis of the expression of an enhancer detector P-element insertion in the stat92E gene and have shown that the level of JAK/STAT pathway activity is high at the midline of the disc and low at both poles. This gradient of pathway activity correlates with the distribution of Upd, which is present at the optic stalk in the center of the eye. Thus, we provide the first evidence that the secreted Upd protein can act at a distance as a developmental patterning signal, activating a gradient of JAK/STAT activity in vivo.

\section{Results}

\section{JAK and STAT are required for correct} ommatidial polarity

LOF clones for mutations in the Drosophila JAK and STAT homologs were generated by the FLP/FRT system (Xu and Rubin 1993). Tangential sections through LOF clones of both hop and stat alleles showed a regular array of ommatidia containing a wild-type complement of correctly differentiated and correctly positioned photoreceptor cells (Fig. 2A,C; data not shown). Thus, the JAK/ STAT pathway is not absolutely required for imaginal disc cell proliferation, cell fate specification, or differentiation. Mutant clones were, however, associated with stereotyped defects in ommatidial polarity.

hop and stat92E LOF clones cause ommatidial polarity inversions

A large proportion of hop LOF clones result in polarity defects in which ommatidia straddling the polar boundary of the clone exhibit inverted DV polarity (Fig. 2A-D). The phenotype is strongest in larger clones and in clones in which the polar boundary runs parallel to the equator. Typically, one or two ommatidial rows are inverted, with the strongest phenotype observed showing about five inverted rows. Mutant ommatidia in the center of the clone and on the equatorial margin of the clone show a normal orientation (Fig. 2A,B). Both totally mutant ommatidia adjacent to the polar boundary and chimeric ommatidia comprising both wild-type and mutant cells on the clonal border can assume an inverted fate. Occasional inversions were observed in clusters immediately outside the clone in which all of the photoreceptors were wild type (arrows in Fig. 2C). LOF hop clones examined in third instar imaginal discs showed the same phenotype (not shown).

We also tested the downstream pathway component STAT by inducing clones of stat92E alleles. These gave qualitatively identical phenotypes to hop clones, but at a lower penetrance (not shown). The frequency with which inversions were recovered was increased in a genetic background heterozygous for hop, demonstrating that removal of a single copy of hop can sensitize the pathway to loss of stat92E. The weak nature of the stat92E phenotype would appear to indicate that the stat92E gene product is only partially required to transduce the hop-mediated signal. Although unexpected,
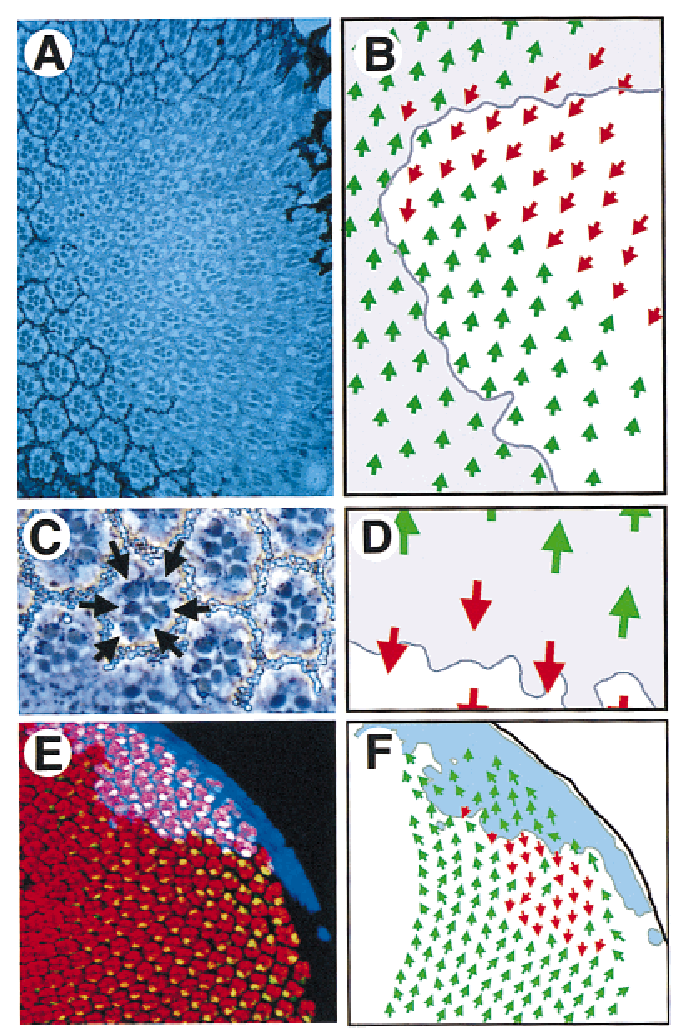

Figure 2. LOF and GOF JAK/STAT pathway clones show abnormal ommatidial polarity. (A) A large dorsal hop clone (marked by absence of white ${ }^{+}$pigment granules) contains normally differentiated ommatidia in both correct and inverted orientation with respect to surrounding wild-type clusters. $(B)$ Schematic representation of the clone shown in A. Dorsal-oriented ommatidia are shown as green arrows and inverted ommatidia are shown in red. The approximate position of the clonal boundary is shown by the purple line. A field of inverted ommatidia is present around the polar margin of the clone. $(C)$ A high-magnification view of ommatidia along the polar boundary of a hop clone (marked by absence of white ${ }^{+}$) showing an inverted ommatidium comprised of entirely wild-type photoreceptor cells (note dark pigment granules associated with the rhabdomeres of wild-type photoreceptors, indicated by arrows). (D) Schematic representation of clone shown in C. Dorsal ommatidia are shown by green and ventral by red arrows. Boundary of the clone is indicated. $(E)$ A clone of cells ectopically expressing Upd (blue) in the dorsal part of an eye disc stained to show Elav (red) and Prospero (green, yellow in overlay). Ommatidia with inverted orientation are seen on the ventral edge of the clone. $(F)$ A schematic representation of clone shown in $E$ indicating the extent of the Upd expressing clone (pale blue) and the ommatidial orientation indicated by dorsal green and ventral red arrows.

this finding is consistent with previous evidence that more than one STAT homolog exists in flies (Sweitzer et al. 1995; Yan et al. 1996b), and suggests that they act semiredundantly in ommatidial polarity determination. Thus, the juxtaposition of wild-type cells and cells unable to transduce the JAK/STAT signal can generate ectopic axes of ommatidial mirror-image symmetry that resemble the normal equator. 
GOF Upd-expressing clones are sufficient to repolarize ommatidia

As LOF JAK/STAT clones result in ectopic axes of ommatidial symmetry, we investigated the effects of ectopic activation of the pathway by misexpression of the pathway ligand Upd. GOF Upd clones were generated by a combination of the FLP/FRT cassette (Struhl and Basler 1993) and GAL4/UAS systems (Brand and Perrimon 1993), such that Upd was expressed in discrete groups of marked cells in the developing eye /colored blue in Fig. 2E). This resulted in inversion of ommatidial polarity in the wild-type tissue on the equatorial side of the clone, with the greatest effect observed in clones closer to the poles of the disc (Fig. 2E,F).

Taken together, these LOF and GOF results indicate that JAK/STAT function across the DV axis of the eye disc is necessary for the normal establishment of a single axis of ommatidial mirror-image symmetry along the DV midline, and is sufficient to define ectopic axes of mirror-image symmetry.

stat92 $\mathrm{E}^{06346}$ as an in vivo reporter of pathway activity

An interesting aspect of the original P-element-mediated insertional mutation in the stat92E locus (stat92E $E^{06346}$, Hou et al. 1996) is the lacZ expression pattern produced by this enhancer detector. Eye discs from larvae carrying this insertion (subsequently referred to as stat92E-lacZ) show a gradient of $l a c Z$ activity that is highest at the poles and decreases to a low point at the DV midline (Fig. $3 \mathrm{~A})$. Increased expression is also seen in the ocellar spot region, and, independently, in many of the macrophagelike blood cells often associated with the eye imaginal disc complex (arrow in Fig. 3A). However, in situ hybridization experiments undertaken with probes specific for the stat92E transcript showed ubiquitous expression of stat92E mRNA in third instar eye discs (not shown), suggesting that this enhancer detector might only report a subset of stat92E transcript expression.

An intriguing possibility was that stat92E-lacZ expression might be related to JAK/STAT pathway activity. We therefore examined stat92E-lacZ staining in larvae carrying the constitutively active $h o p^{\text {Tuml }}$ allele of Drosophila JAK (Hanratty and Dearolf 1993). In hop ${ }^{\text {Tuml }}$ eye discs with uniformly increased JAK/STAT activity, the overall level of lacZ activity was consistently lower (Fig. 3C) than in discs from wild-type siblings stained in parallel (Fig. 3B). To confirm this apparent negative regulation of stat92E-lacZ expression by the JAK/STAT pathway, we carried out two further complementary experiments. Firstly, we induced clones lacking hop in a stat92E-lacZ background. In such clones lying close to the equator, a strong cell autonomous upregulation of stat92E-lacZ was observed to a level comparable with that seen at the poles (Fig. 3D-F). Secondly we ectopically activated the JAK/STAT pathway to higher levels than could be achieved using the hop ${ }^{\text {Tuml }}$ allele by ectopically expressing Upd in the presence of the stat92ElacZ reporter. Clones of cells expressing Upd close to the

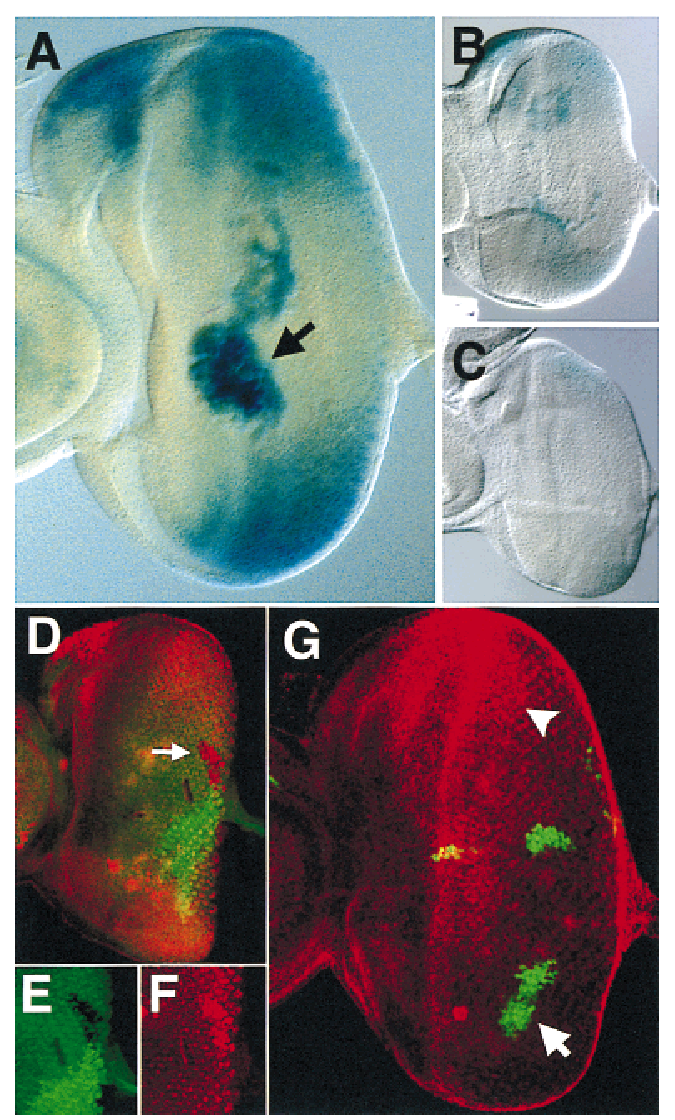

Figure 3. The expression of stat92E-lacZ is negatively regulated by the activity of JAK/STAT signaling. $(A)$ stat92E-lacZ staining in a third instar disc showing highest levels of lac $Z$ at the poles of the eye field, at the ocellar spot, and in macrophage cells located in the middle of the eye disc below the plane of focus (arrow). $(B, C)$ Eye discs prepared and stained under identical conditions carrying stat $92 E-1 a c Z(B)$ and stat92E-lacZ in a hop ${ }^{\text {Tuml }}$ mutant background $(C)$. $(D-F)$ Double label of eye imaginal disc stained for stat92E-lacZ (red) and a GFP clonal marker (green). A hop ${ }^{2}$ clone marked by lack of green staining (arrow in $D$, enlarged in $E$ ) and results in higher levels of stat92E-lacZ expression autonomously within the clone $(D$, enlarged in $F$ ). (G) Upd and GFP (green) are coexpressed in GOF clones within the eye imaginal disc also stained to show the pattern of stat92E-lacZ (red). An Upd-expressing clone in the ventral portion of the disc (arrow) completely abolishes the stat92E-lacZ gradient in the adjacent tissue such that no staining is seen at the ventral pole. Staining at the dorsal pole remains strong (arrowhead). Large cells staining in the middle of the disc are macrophages.

equator of the disc had no effect (Fig. 3G and data not shown), however clones close to the poles (arrow Fig. 3G) resulted in complete loss of polar stat92E-lacZ activity in that hemisphere, although normal levels of stat92Elac $Z$ were observed at the opposite pole (arrowhead Fig. 3G).

We have, therefore, shown that the level of stat92Elac $Z$ expression is inversely proportional to the level of JAK/STAT pathway activation: High activation produced by Upd expression abolishes stat92E-lacZ activ- 
ity, moderate activation produced by the hop ${ }^{\text {Tuml }}$ allele gives reduced activity, whereas cells in which there is no JAK/STAT signaling (such as hop clones) show maximal levels of stat92E-lac $Z$ activity.

Comparing the results of these experiments with the endogenous pattern of stat92E-lacZ staining in the eye disc, we conclude that JAK/STAT activity must be highest at the DV midline (where stat92E-lacZ activity is lowest) and low at the poles (where stat92E-lacZ activity is upregulated to levels similar to those seen in hop clones) with a gradient of JAK/STAT activity present between these extremes (as visualized by the equatorial/ polar gradient of stat92E-lacZ expression, Fig. 3A).

\section{Upd is expressed on the DV midline}

Given the known function of Upd as a JAK/STAT pathway ligand during Drosophila embryonic development, and our results showing that expressing Upd during eye development can mimic activation of the JAK/STAT pathway, we decided to investigate whether Upd was likely to be acting as the endogenous JAK/STAT pathway ligand during eye patterning.

Therefore, we analyzed the time course of Upd expression during eye development, using a polyclonal antiUpd antibody (Harrison et al. 1998). In first instar discs, only very weak expression was observed, in a faint horseshoe-shaped pattern around the poles and posterior of the disc (Fig. 4A). By second instar, expression was seen localized to the posterior margin of the disc, lying on the DV midline adjacent to the optic stalk (Fig. 4B). This pattern of expression persisted into the third instar stage (Fig. 4C-E), however, by late third instar, an additional patch of staining was seen at the anteroventral margin of the eye disc adjacent to the junction with the antennal disc (Fig. 4C). Higher magnification views of an early third instar disc reveal that the Upd protein is highly expressed in only a small group of cells on the DV midline, where it can be seen cytoplasmically localized (Fig. 4D), but consistent with it being a secreted ligand, the protein is seen around the periphery of cells away from the site of expression in a concentration gradient toward the poles of the disc (Fig. 4D,E).

Considering the expression domain of endogenous Upd in the eye and the negative regulation of stat92ElacZ by ectopically expressed Upd, it seems likely that the wild-type pattern of stat92E-lac Z is at least partly a consequence of endogenous Upd expression. We note that the expression pattern of stat92E-lacZ in the third instar disc does not seem to be the exact inverse of the Upd expression pattern. However, it must be borne in mind that it is difficult to predict the exact stat92E-1acZ pattern that would be expected, because stat92E-lacZ is probably a lagging indicator of Upd repression (due to the perdurance of the $\beta$-galactosidase gene product) and the cells in the posterior of the disc (behind the furrow) undergo fewer cell divisions, which would tend to distort the pattern seen.

On the basis of this evidence, we propose that the Upd expression at the optic stalk is sufficient to set up a gra- dient of JAK/STAT activation across the DV axis of the developing eye disc.

Ectopic expression of Upd at the poles
repolarizes the eye

Further experiments were carried out to test the hypothesis that localized expression of Upd at the DV midline and the consequent gradient of JAK/STAT expression is important for normal eye patterning. The normal pattern of Upd expression was altered by ectopically expressing Upd in the developing eye imaginal disc using the GAL4/UAS system and the 30A-GAL4 line, which drives expression at the dorsal and ventral poles of the developing eye disc (Brand and Perrimon 1993). In wildtype eyes, the array of facets appears externally regular (Fig. 4F), however, misexpression of Upd at the poles of the eye gave rise to adults in which the normal regular array of ommatidial facets was externally disrupted at both the dorsal and ventral poles of the eye (arrow in Fig. $4 G$; data not shown). Sections through either the dorsal or ventral regions of such eyes showed that although equatorially ommatidial polarity was normal, at the poles (where Upd was ectopically expressed) ommatidia had inverted orientation (Fig. 4H,I). Note that the inversions generated by misexpressing Upd in this manner do not form a regular and straight equator but rather appear to define a field of inversion with no clear boundary between dorsal and ventral ommatidial fates. This confused region may well represent the area in which ommatidia are responding to competing polarity signals, produced either by endogenous Upd expression at the optic stalk and Upd at the poles or possibly by other independent signaling mechanisms (see Discussion).

Loss of upd at the optic stalk results in dorsalization of the eye field

Next, we investigated the effect of completely removing Upd expression from the developing eye. Clones homozygous mutant for two independent Upd alleles were generated. These were recovered at high frequency and all of the clones analyzed, which lay within the eye field, displayed no visible phenotype either in imaginal discs or adult eyes (not shown). However, clones that overlapped the region of Upd expression adjacent to the optic stalk produced almost complete dorsalization of the eye field as assayed by ommatidial polarity (Fig. 5A-C).

In an effort to better understand the ommatidial dorsalization observed following removal of Upd, we looked at the expression of mirr. Wild-type mirr expression is restricted to the dorsal hemisphere of the eye (Fig. 5D,I; McNeill et al. 1997), and via repression of fng results in activation of the Notch pathway at the DV midline thereby defining the position of the endogenous equator (Cho and Choi 1998; Domínguez and de Celis 1998; Papayannopoulos et al. 1998). To investigate mirr regulation, we used P-element insertions in mirr that function as enhancer detectors and express both the lac $Z$ gene and 


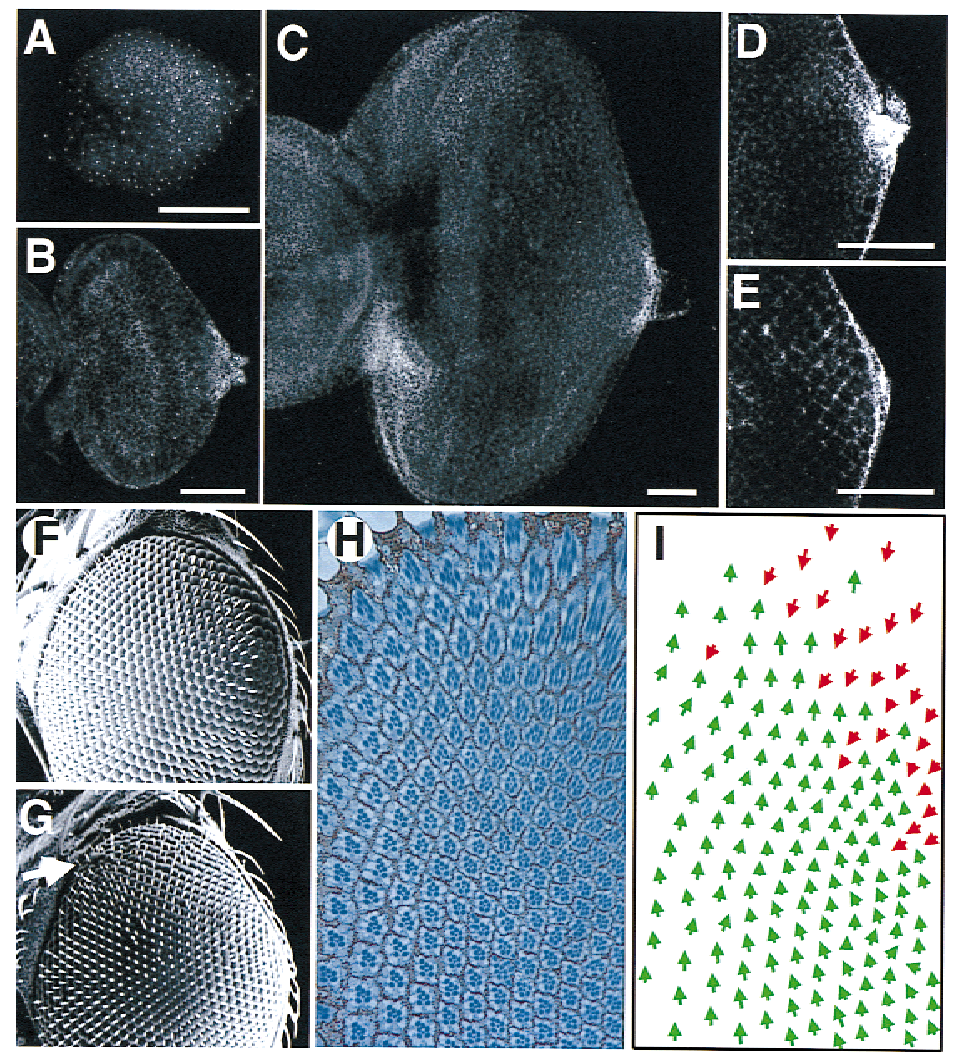

Figure 4. The wild-type expression pattern of Upd and the phenotype induced by ectopic expression of Upd from the poles of the eye. $(A-E)$ The pattern of staining with an anti-Upd polyclonal antibody; scale bars, $50 \mu \mathrm{m}$. (A) First instar disc. Staining is faintly visible in a horseshoe around the poles and posterior margin of the disc. The small bright spots of staining distributed across the whole disc may represent staining in the peripodial membrane and/or could be nonspecific. (B) Second instar. Expression is localized to the posterior margin at the DV midline, adjacent to the optic stalk. $(C)$ Late third instar. The furrow has progressed more than halfway across the disc. Expression is still seen localized adjacent to the optic stalk, however, a new region of high-level expression is evident on the ventro-anterior margin adjacent to the antennal disc, which is not present in younger discs (and thus presumably plays no role in ommatidial polarity determination). ( $D$ ) Higher magnification view of the posterior midline region of an early third instar disc, focused basally. A small cluster of cells shows high-level cytoplasmic expression. (E) More apical view of disc shown in $D$. A gradient of extracellular Upd can be seen in the extracellular space between the nascent ommatidial clusters. $(F)$ Scanning electron micrograph (SEM) of the dorsal half of a wild-type adult eye. $(G)$ SEM of the dorsal half of an eye in which Upd expression was driven at the dorsal and ventral poles during development by the 30A-GAL4 driver at $18^{\circ} \mathrm{C}$. The eye appears normal apart from a weak disruption of externally visible facet packing at the dorsal pole (arrow). ( $H$ ) Section through the dorsal region of an eye similar to $G$ produced by expression of Upd with the 30A-GAL4 driver. The eye is normal in the ventral part of the field (close to the equator), but inverted ommatidia are seen near the dorsal pole. (I) Schematic representation of $C$. Dorsal ommatidia are shown by green and ventral by red arrows. Note that there is a region of inversion of ommatidial polarity near the dorsal pole, but that ommatidia of both fates are clearly mixed.

the P-element mini-white $e^{+}$marker gene specifically in the dorsal half of the adult eye, henceforth referred to as mirr-lacZ.

When mirr-lac Z was present in a background in which clones removing upd were generated, a low frequency of flies were recovered in which all or almost all of the eye field expressed the dorsal fate-specific white ${ }^{+}$mirr reporter (Fig. 5E). When the eyes of flies showing this external dorsalization phenotype were sectioned, the dorsal fate of the ommatidia within the region ectopically expressing mirr was confirmed (Fig. 5F,G), although intriguingly, such eyes showed occasional ommatidia still exhibiting ventral fate in the mirr-expressing region (shown as red arrows in the gray-shaded region in Fig. $5 \mathrm{G})$.

Therefore, we conclude that Upd expression at the optic stalk during normal eye patterning is required for restriction of mirr expression to the dorsal hemisphere of the eye. This restriction then determines the position of the equator along the DV midline of the eye disc via activation of Notch.

\section{IAK/STAT LOF clones produce polarity inversions via a mirr-independent mechanism}

Given the role of Upd in restricting mirr expression, one possible mechanism by which JAK/STAT LOF clones might induce ectopic axes of mirror-image symmetry would be through the generation of ectopic boundaries of mirr expression. We therefore analyzed the expression of mirr-lacZ in hop clones. Many clones lying both dorsally and ventrally were examined in eye discs, and in no case was an alteration in mirr-lac $Z$ expression observed (Fig. 5H-J and data not shown). Additionally, hundreds of adults carrying mirr-lac $Z$ were examined, in which hop clones had been induced, and, again, in no case was a change in mirr-regulated white $e^{+}$expression observed.

Thus, ommatidial polarity inversions generated by hop clones are mirr independent. We therefore conclude that the process of midline equator definition by dorsally restricted mirr expression and the regulation of ommatidial polarity by the JAK/STAT pathway are separable processes. We also note that our results suggest that Upd might act independently of Hop to regulate mirr expression, although we cannot exclude the possibility that we never obtained a hop clone that exactly overlapped the domain of Upd expression.

The relationship of the Upd/JAK/STAT pathway to the Wg pathway in ommatidial polarity determination

Our results show that LOF JAK/STAT clones give nonautonomous inversions of ommatidial polarity on the 


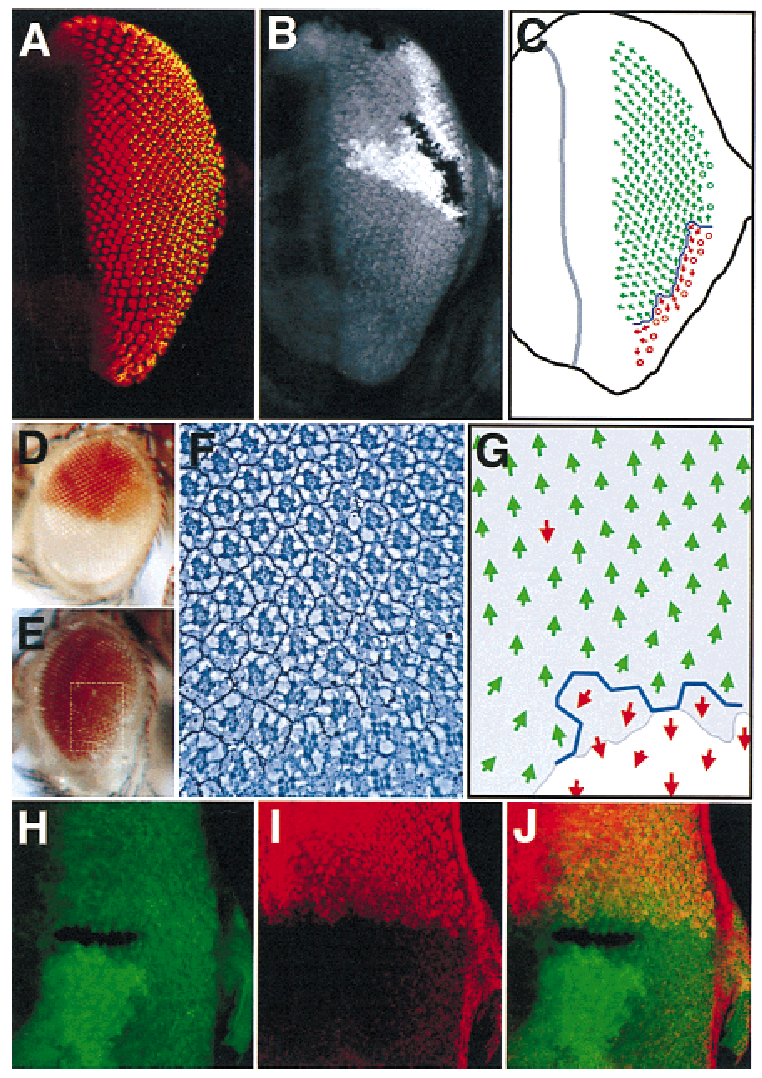

Figure 5. Upd is required for dorsal restriction of mirr expression and positioning of the equator, but hop clones do not affect mirr expression. $(A, B)$ An eye disc containing an Upd clone situated at the posterior of the eye field is dorsalized with the equator shifted to the ventral margin. (A) The photoreceptor markers Elav (red) and Prospero (green, yellow in overlay); $(B)$ The clonal marker in which nonstaining cells lack upd function. $(C)$ Schematic representation of the disc shown in $A$ and $B$. Much of the eye field has assumed the dorsal (green arrow) fate and only a small number of ventral ommatidia (red arrows) are present along the ventral posterior margin of the eye separated by an equator (blue line) (O). Ommatidia with unscoreable rotation. (D) A wild-type eye showing the dorsal white ${ }^{+}$expression domain of a mirr enhancer-detector P-element insertion. (E) mirr enhancer-detector expression throughout the eye of a fly in which upd LOF clones have been induced. A slight reduction in eye size and disorder of the ommatidial array in ventral regions is apparent. $(F)$ Section through an eye similar to that shown in $E$ showing the expression domain of the mirr enhancer-detector white (dark pigment granules). The approximate area sectioned is indicated by the dotted white box in $E$. Note that this particular eye had a small region of white $e^{-}$tissue at the ventral edge, which shows normal ventral ommatidial orientation. $(G)$ Schematic representation of $F$ showing the ommatidial orientation with dorsal (green arrow) and ventral (red arrow) fates. Shading indicates the extent of mirr expression (as revealed by regulation of white $\left.{ }^{+}\right)$. Note that some ommatidia in the mirr-expressing region nevertheless show ventral rotational fate. $(H-J)$ Ventral hop clones do not activate mirr expression. Confocal image of an eye disc containing a ventrally situated hop clone marked by lack of green staining $(H, J)$ also stained for dorsal-specific expression of a mirr enhancer detector in red $(I, J)$. polar clonal boundary, and that JAK/STAT activity is highest at the DV midline as a result of localized Upd expression. This situation is reciprocal to that seen for LOF Wg pathway clones that cause nonautonomous inversions of ommatidial polarity on the equatorial clonal boundary (Wehrli and Tomlinson 1998). An important question is whether the $\mathrm{Wg}$ and JAK/STAT pathways are acting independently to regulate ommatidial polarity decisions, or whether one acts through the other.

We therefore carried out experiments to test directly whether Upd and Wg might regulate each other's expression. We found that ectopic Upd expression had no effect on Wg expression in the developing eye disc (Fig. 6A) and also that ectopic expression of $\mathrm{Wg}$ adjacent to the optic stalk did not alter Upd expression (Fig. 6B). Thus, Upd cannot be producing its phenotype via negative regulation of $\mathrm{Wg}$ and, similarly, $\mathrm{Wg}$ does not act via regulation of Upd.

These results indicate that Wg and Upd do not regulate each other's expression, and, thus, that one of these pathways is not likely to be downstream of the other. Instead, we surmise that Wg and Upd act in parallel to one another in the regulation of ommatidial polarity.

\section{Discussion}

In this paper we present data showing a specific role for the JAK/STAT signal-transduction pathway in the developing Drosophila eye. We demonstrate that a gradient of JAK/STAT activation is required for normal polarity determination on the DV axis, and that localized expression of the secreted ligand Upd at the DV midline is capable of setting up such a gradient. We also show that ectopic expression of Upd alone is able to induce ectopic axes of ommatidial symmetry. Finally, we find an additional role of upd in defining the position of the equator via regulation of mirr expression.
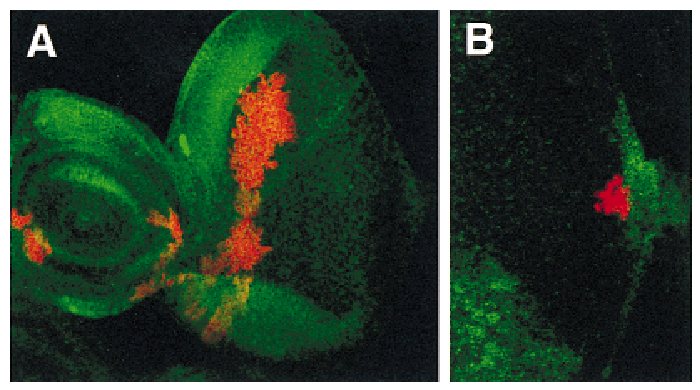

Figure 6. Wg and Upd do not regulate each other's expression. (A) A disc containing clones of cells ectopically expressing Upd (red), stained for expression of Wg protein (green). Strong Wg expression can be seen at the dorsal margin, adjacent to a large patch of Upd-expressing cells, indicating that Upd does not repress $\mathrm{Wg}$ expression. $(B)$ The posterior region of a disc containing clones of cells ectopically expressing $\mathrm{Wg}(\mathrm{red})$, also stained for Upd expression (green). Upd expression remains at the optic stalk even when adjacent to Wg-expressing cells. 
JAK/STAT signaling affects ommatidial polarity via a second signal

The ommatidial polarity phenotype produced by removal of JAK activity in mosaic clones has a number of important features. Firstly, the phenotype observed is an inversion of ommatidial polarity in which either the dorsal rotational form is seen in the ventral hemisphere of the eye or vice versa. Secondly, the phenotype is only observed on the polar boundary of the mosaic tissue. Thirdly, the strength of the phenotype (in terms of the number of inverted ommatidia seen) is dependent on the size and shape of the clone. Fourthly, the phenotype is cell nonautonomous as either fully mutant, fully wildtype, or mosaic clusters can manifest the phenotype.

From these characteristics, we can deduce the following: The nonautonomy of the phenotype produced by removal of the autonomously acting pathway component JAK, and its dependence on clone size and shape, suggests that JAK/STAT affects ommatidial polarity via a secreted downstream signal (which we will subsequently refer to as a second signal). The direction of the nonautonomy (only in a polar direction) and the strict DV nature of the polarity inversions indicates that this second signal must be graded in its activity along the DV axis, with a change in direction of the gradient at the equator. The direction of this gradient would then be the instructive cue to which ommatidia respond when rotating to establish their mature polarity.

As we have demonstrated that JAK/STAT activity itself is graded along the DV axis, with highest activity at the midline, the simplest interpretation of these results is that JAK/STAT signaling positively regulates the secretion of a second signal, which is highest at the equator and lowest at the poles. This then explains why JAK/ STAT LOF function clones produce inversions of ommatidial polarity only on the polar side of the clone-the predicted diffusion of the second signal into the hole made by the clone will produce an ectopic peak of second signal just outside of the polar boundary of the clone that phenocopies the normal equator (Fig. 7A,B). Similarly, Upd GOF clones are predicted to produce equatorial inversions (Fig. 7C,D).

Only a model such as this, in which the expression of the second signal (which is the primary determinant of ommatidial polarity) is proportional to JAK/STAT activity can explain the ommatidial polarity inversions that we see on the polar side of LOF clones and the equatorial side of GOF clones. The simpler hypothesis that a single threshold level of JAK/STAT activity defines the region of second signal secretion (i.e., at the DV midline) does not explain why LOF clones near the poles of the eye disc (where JAK/STAT activity is always low) can induce ectopic equators.

Whereas in our diagrams we have represented the second signal as being at high levels at the equator and low levels at the poles, it is equally likely that the gradient of the second signal is in fact highest at the poles of the disc and lowest at the equator, with Upd acting as a repressor of second signal expression. Whereas the molecular nature of the second signal (or signals) remains unknown, these alternatives are impossible to distinguish.

A similar gradient model involving a second signal has recently been proposed to explain the phenotypes observed for LOF and GOF clones of the Wg signal-transduction pathway. An important difference is that $\mathrm{Wg}$ is expressed in the polar regions of the eye disc and is predicted to be a repressor of the second signal ( $\mathrm{Ma}$ and

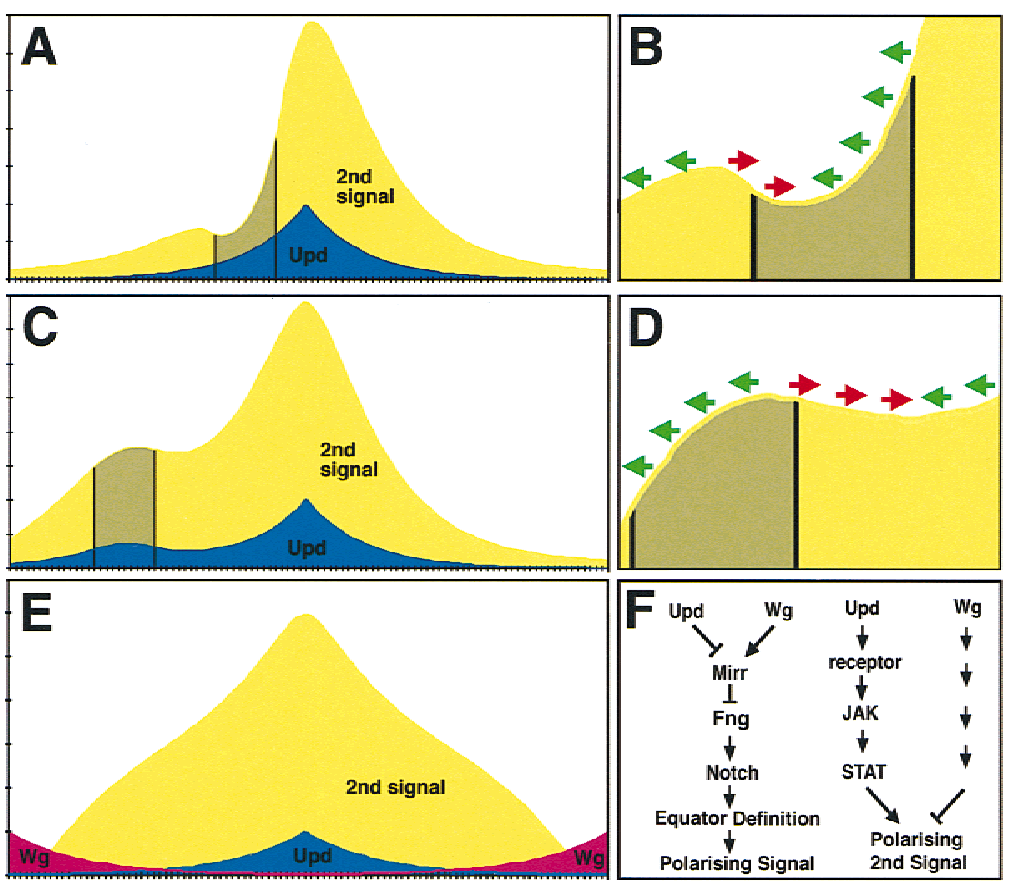

Figure 7. A model for how Upd/JAK/STAT signaling determines ommatidial rotational fate. $(A-E)$ Graphs that represent a single dorsal (left) to ventral (right) line of cells through an eye imaginal disc. The levels of Upd (blue) and second signal (yellow) are shown with the peak of Upd corresponding to the equator or center of the disc. (Green arrows) Dorsal rotational fate; (red arrows) ventral fate $(B, D) .(A, B)$ The effect of a JAK/STAT LOF clone (gray) on ommatidial polarity; $(C, D)$ the effect of an Upd misexpressing clone. $(E)$ The predicted concentration of a single second signal if it were both negatively regulated by $\mathrm{Wg}$ diffusing from the poles and positively regulated by Upd from the equator; note that the opposing effects of shallow Upd and Wg gradients can combine to produce a steep gradient of second signal. $(F)$ The two proposed functions of Upd/JAK/ STAT in ommatidial polarity determination: Upd acts together with Wg to restrict mirr expression and define the position of the equator via activation of Notch, which itself leads to production of an ommatidial polarizing signal; Upd also activates a gradient of JAK/STAT activity that indirectly regulates ommatidial polarity by a mirr-independent mechanism; additionally, Wg secreted from the poles also produces a gradient of an ommatidial polarizing signal. 
Moses 1995; Treisman and Rubin 1995; Reifegerste et al. 1997; Heberlein et al. 1998; Wehrli and Tomlinson 1998), such that Wg GOF clones behave like JAK/STAT LOF clones showing polarity inversions on the polar clonal boundary.

As we have demonstrated that Upd expression does not regulate $\mathrm{Wg}$ and vice versa, we can exclude the possibility that these two pathways act sequentially, and so propose that they must act in parallel. An attractive possibility is that the Upd and Wg pathways might act in parallel to regulate the concentration of a single second signal. In this case, Upd expression at the DV midline would activate the signal and Wg expression at the poles would repress the signal. Adding together the effect of two such opposing signals produces a predicted second signal concentration that has a fairly even slope from the DV midline to the poles (Fig. 7E). In contrast, a single signal that is high at the DV midline and decays to zero at the poles has a very shallow gradient in the polar regions (cf. Fig. 7A). As reading the slope of a steep gradient is presumably easier than reading the slope of a shallow gradient, the use of two opposing gradients to set up second signal concentration is thus highly advantageous. Therefore, this presents a possible biological explanation for the proposed redundant use of both an Upd and a Wg concentration gradient in determining ommatidial polarity.

\section{The interpretation of the second signal}

The most likely candidate for a receptor of the second signal is the seven pass transmembrane protein encoded by the frizzled $(f z)$ locus (Vinson et al. 1989). $f z$ function is required in the presumptive R3/4 cells of the pre-ommatidium, and clonal analysis suggests that it interprets a gradient of positional information that is high at the equator and low at the poles (Zheng et al. 1995). Recent results suggest that differential activation of $\mathrm{Fz}$ signaling in R3/4 results in asymmetric Notch activation in this photoreceptor pair, which ultimately leads to a binary cell-fate decision such that the cell closest to the equator takes on the R3 fate and the ommatidial unit as a whole adopts the correct rotational fate (Cooper and Bray 1999; Fanto and Moldzik 1999).

\section{Localized Upd expression and definition of the equator}

Three recent studies have demonstrated a critical role of Iroquois family transcription factors such as Mirr in positioning the normal equator (Cho and Choi 1998; Domínguez and de Celis 1998; Papayannopoulos et al. 1998). Dorsally restricted mirr expression represses the expression of $f n g$, which is consequently restricted to the ventral hemisphere. Thus, mirr and fng expression abut at the DV midline, and this leads to activation of Notch in a narrow band of cells. This localized activation of Notch is then sufficient to define the equator and organize ommatidial polarity, apparently through secretion of an unidentified second signal.
Our results show that localized Upd expression on the DV midline at the posterior margin is also necessary for the correct positioning of the equator, and that when upd function is removed from this region by clones induced during early first instar development, the eye field becomes dorsalized and the equator is shifted to the ventral edge of the eye. This shift in the position of the equator coincides with ectopic expression of the homeobox gene mirr in the ventral hemisphere. Thus, we propose that Upd acts upstream of the mirr/fng/Notch-dependent mechanism for definition of the equator, by either directly or indirectly regulating mirr expression.

We note that this role of Upd in positioning the equator on the DV axis is reciprocal to the function of $\mathrm{Wg}$ in this process. Overexpression of $\mathrm{Wg}$ from the polar regions causes the equator to be shifted toward the ventral margin of the disc, as assayed both by ommatidial polarity and by expression of mirr (Heberlein et al. 1998). Conversely, removal of $w g$ function causes at least partial ventralization as mirr expression is lost. Thus in the dorsal hemisphere, Wg activates mirr expression, whereas in the ventral hemisphere, Upd represses it.

Interestingly, the Unpaired expression domain, located at the posterior midline of the disc, coincides with the position at which the morphogenetic furrow is initiated. Furthermore, previous reports have linked furrow initiation with ommatidial rotation /Chanut and Heberlein 1995; Strutt and Mlodzik 1995; Reifegerste et al. 1997). Thus, it is conceivable that Upd expression might actually determine the site of furrow initiation, and that Upd GOF clones might induce ectopic furrow initiations. However, Upd LOF clones that overlap the normal expression domain of Upd still result in furrow initiation (Fig. 5A-C) and, similarly, in GOF clone experiments we saw no evidence for ectopic furrow initiation events. Therefore, we currently have no evidence to support this hypothesis.

\section{Regulation of ommatidial polarity by JAK/STAT signaling is mirr independent}

Although Upd acts as both a regulator of mirr expression and an activator of the JAK/STAT pathway, we found no evidence that mirr is itself regulated by JAK/STAT. No change in mirr expression in discs or adults eyes was observed in hop LOF clones in either the dorsal or ventral hemisphere. Therefore, the ommatidial polarity inversions observed on the polar edges of hop clones cannot be produced as a consequence of inducing an ectopic mirr/fng boundary.

We note that our results only indicate a role for Upd function as a repressor of mirr expression in the ventral hemisphere of the eye. However, JAK/STAT LOF clones in both the dorsal and ventral hemispheres result in ommatidial polarity defects. This again supports a mirr-independent function of the JAK/STAT pathway in ommatidial polarity determination.

A similar situation appears to exist in the case of the Wg pathway. Wg itself is required as an activator of mirr expression only in the dorsal hemisphere (Heberlein et 
al. 1998), however, Wg-pathway LOF clones in both the dorsal and ventral hemispheres have been reported to result in reorganization of ommatidial polarity (Wehrli and Tomlinson 1998). It is not currently known whether Wg pathway LOF clones in the eye result in changes in mirr expression. However, by analogy to Upd/JAK/ STAT function, it is possible that Wg both acts to position the equator via a mirr-dependent mechanism and also regulates ommatidial polarity in both the dorsal and ventral hemispheres by a mirr-independent mechanism.

The reorganizations of ommatidial polarity observed when Upd is ectopically expressed are almost certainly a consequence of ectopic activation of JAK/STAT signaling, rather than any effect on mirr expression. This follows from the fact that Upd misexpression produces the same phenotypes both dorsally and ventrally, whereas Upd only represses mirr ventrally.

\section{Redundancy between JAK/STAT and mirr/fng/Notch pathways}

There is now overwhelming evidence that the polarity of ommatidia is determined by the slope of the gradient(s) of one or more unidentified diffusible second signals. Furthermore, the concentration of the second signal(s) appear to be dependent on Wg pathway activity, JAK/ STAT pathway activity, and Notch activity at the DV midline. Given our present state of knowledge, it is possible that each of these three signaling pathways could be regulating either the same or different second signals.

The simplest model would be that there is a single second signal secreted from the equator, which is downstream of mirr/fng/Notch, and that Wg and Upd/JAK/ STAT feed into this pathway upstream of Notch. This is consistent with the roles of $\mathrm{Wg}$ and $\mathrm{Upd}$ as regulators of mirr expression and, thus, in positioning the endogenous equator. However, it is not consistent with the observed ommatidial polarity inversions produced in the eye field both dorsally and ventrally by Wg-pathway and JAK/ STAT-pathway LOF and GOF clones. These phenotypes indicate that second-signal concentration is dependent on Wg pathway and JAK/STAT pathway activity across the whole of the eye field (see above), and thus the second signal cannot be only secreted from the DV midline as a consequence of localized Notch activation. It is conceivable that Notch is activated on the polar boundary of JAK/STAT LOF clones, but in this context the only known mechanism of Notch activation is via mirr/fng interactions, and we have ruled out this possibility.

Instead, our data points to a model in which Upd and Wg first act to define the equator via restriction of mirr expression to the dorsal hemisphere and localized activation of Notch along the DV midline. Definition of the equator is known to occur early in development (Cho and Choi 1998), while the disc is still small, and divides the disc into two hemispheres separated by a straight boundary that will form the future equator. Such boundaries evidently serve as a source of a second signal that can polarize ommatidia, as $f$ g LOF clones that induce ectopic regions of activated Notch result in changes in ommatidial polarity (Cho and Choi 1998; Domínguez and de Celis 1998; Papayannopoulos et al. 1998).

Subsequently in development, we surmise that gradients of JAK/STAT and Wg-pathway activity across the DV axis of the eye disc are responsible for setting up a gradient(s) of one or more second signals that can determine ommatidial polarity. These signals might be responsible for maintaining longer range polarization of ommatidia away from the equator and the localized Notch-dependent polarizing signal.

There is a great deal of support for such a model. First, it is consistent with the known timing of the events involved. The requirement for fng function has been shown to lie between late first instar and mid second instar (Cho and Choi 1998), which coincides with the first appearance of high levels of Upd expression at the optic stalk. However, the ommatidia are not formed (and thus do not respond to the polarity signal) until the start of the third instar, a stage when localized Upd expression still persists. Furthermore, extracellular Upd protein can be seen in a concentration gradient many cell diameters from the optic stalk at the early third instar stage, consistent with Upd being at least partly responsible for setting up the long-range gradient of JAK/STAT activity across the DV axis of the eye disc that is revealed by the stat92E-lacZ reporter.

Secondly, this model does not require that a single source of second signal secreted by a narrow band of cells at the equator should be capable of determining ommatidial polarity across the whole of the DV axis of the disc during the third instar stage of development. Instead, the band of activated Notch at the equator would serve to draw a straight line between the fields of dorsally and ventrally polarized ommatidia, and need only secrete a localized source of second signal to polarize ommatidia in this region. Further from the equator, the opposing gradients of Upd and $\mathrm{Wg}$ signaling would provide a robust mechanism for maintenance of correct ommatidial polarity across the DV axis. Conversely, without the mirr/fng/Notch mechanism to draw a straight line, it would be impossible to imagine how Upd at the posterior margin and $\mathrm{Wg}$ at the poles alone could provide the perfectly straight equator that is ultimately formed.

Thirdly, the phenotypes that we observe are consistent with multiple competing mechanisms responsible for determining ommatidial polarity. When we induce inversions of ommatidial polarity by generating hop clones or ectopically expressing Upd, we do not produce straight equators, such that we obtain two cleanly abutting fields of dorsal and ventral ommatidia (Fig. 2A,B,E,F; Fig. $4 \mathrm{H}, \mathrm{I})$. Instead, there is usually some confusion of ommatidial identities as if they might be receiving conflicting signals. Additionally, when upd activity is removed from the optic stalk, an equator still forms (albeit at the ventral edge of the disc), but some ommatidia dorsal to the equator still adopt a ventral fate (Fig. 5F,G) as if the determination of ommatidial polarity is less robust in the absence of Upd. 


\section{Concluding remarks}

This is the first report of a developmental patterning role for a gradient of JAK/STAT activity during Drosophila development and of the ability of the Upd secreted protein to set up such a signaling gradient at a distance from its site of expression.

Upd is required to position the future equator via dorsal repression of mirr and localized activation of Notch at the DV midline, and also appears to regulate ommatidial polarity via activation of a gradient of JAK/STAT pathway activity and secretion of an unidentified second signal. Intriguingly, in both of these contexts, Wg secreted from the poles of the disc appears to cooperate with Upd. It will be interesting to see whether this represents a general mechanism for cooperation of these signaling pathways in pattern formation.

\section{Materials and methods}

Fly stocks

Fly stocks used include the following: hop ${ }^{4}$ and hop ${ }^{2}$ (known previously as hop ${ }^{H C 257}$ and hop ${ }^{C 111}$, respectively; Perrimon and Mahowald 1986); stat92E $E^{06346}$ (Hou et al. 1996) and stat92E ${ }^{j 6 C 8}$ (Berkeley Genome Project); upd[ $\left.{ }^{o s 1 A}\right]$ and upd (known previously as asupd ${ }^{Y M 55}$; Harrison et al. 1998.) Upd is also known as outstretched and sisterless-C. The mirr-lacZ reporters B1-12 (Choi et al 1996) and 1(2)f7 (Brodsky and Steller 1996) were also used. The FRT18A and FRT82B lines (Xu and Rubin 1993) were either recombined with the mutations listed above or the $P[U$ biquitin-GFP, $\left.W^{+}\right]$(Davis et al. 1995) and $P\left[\mathrm{~W}^{+}\right]$markers. Clones were induced by two $3-\mathrm{hr} 38^{\circ} \mathrm{C}$ heat shocks at $24-48$ and $48-72$ hr of development to express the FLP recombinase (Golic and Lindquist 1989). hop ${ }^{\text {Tuml }}$ (Hanratty and Dearolf 1993) interaction with stat92E-lacZ was visualized after 3 days at $29^{\circ} \mathrm{C}$. Larvae hemizygous for hop ${ }^{\text {Tuml }}$ were selected by the presence of melanotic tumors.

A recombinant of $p\left[A c t>y+>G a l 4, w^{+}\right]$with cytoplasmic $p\left[U A S-G F P, w^{+}\right]$(Ito et al. 1997) was used to generate and mark GOF clones. UAS-Wg (gift of Henry Krause, University of Toronto, Toronto, Ontario, Canada) and UAS-Upd were crossed to $p\left[A c t>y^{+}>G a 14, w^{+}\right], p\left[U A S-G F P, w^{+}\right]$in the presence of a FLP source; $24-48 \mathrm{hr}$ larvae were heat shocked for $45 \mathrm{~min}$ at $37^{\circ} \mathrm{C}$ followed by incubation at $18^{\circ} \mathrm{C}$ (UAS-Wg) or $25^{\circ} \mathrm{C}$ (UAS-Upd) until mid-third instar. UAS-Upd was generated and transformed into $W^{1118}$ flies by standard methods.

\section{Histology}

Eye imaginal discs were dissected from wandering third instar larvae, fixed in PBS $+6 \%$ formaldehyde ( $2 \%$ for anti-Upd) for 15-20 min, and stained with rat anti-Elav (Robinow and White 1991) at 1:40; mouse anti-Prospero (Spana and Doe 1995) at 1:3; Rat anti-Upd (Harrison et al. 1998) at 1:1000. Rabbit anti-LacZ (Cappel) was preabsorbed and used at a final concentration of 1:4000. Fluorescently conjugated secondary antibodies (Jackson labs) were used at 1:250 to 1:500 dilution. Anti-LacZ was visualized with HRP-conjugated anti-rabbit (Vector Laboratories) 1: 1000, the TSA-Indirect amplification kit (NEN Lifescience) and streptavidin-Texas red (1:500). All washes used PBS plus $0.1 \%$ Triton X-100 except for anti-Upd, which used PBS plus $0.1 \%$ Tween 20. All discs were postfixed in PBS plus $4 \%$ formaldehyde prior to mounting and visualization on a Leica TCS NT confocal microscope. LacZ activity staining was visualized as described in Brand and Perrimon (1993). Adult eye sections (1-2 $\mu \mathrm{m}$ ) were prepared by standard methods (Tomlinson and Ready 1987); heads for S.E.M. were critical-point dried and coated with $25 \mathrm{~nm}$ of gold by standard methods prior to imaging.

\section{Acknowledgments}

We thank Herman Steller, the Berkeley Drosophila Genome Project and Bloomington for fly stocks, Eric Spana, Susan Parkhurst, and Doug Harrison for antibodies, and Doug Harrison and Kwang Choi who kindly shared results prior to publication. Lutz Kockel and members of the Perrimon laboratory provided helpful feedback during the course of this project. We thank Susan Smith and Helen Strutt for comments and Chuck Arnold for the generation of transformant lines and John Proctor for assistance with scanning electron microscopy. M.Z. was supported by European Molecular Biology Organization and The Human Frontiers Science Program long-term postdoctoral fellowships, N.P. is a Howard Hughes Investigator, and D.S. is a Lister-Jenner Research Fellow with additional support from the Wellcome Trust and the Medical Research Council.

The publication costs of this article were defrayed in part by payment of page charges. This article must therefore be hereby marked 'advertisement' in accordance with 18 USC section 1734 solely to indicate this fact.

\section{References}

Baker, N.E. and G.M. Rubin. 1992. Ellipse mutations in the Drosophila homologue of the EGF receptor affect pattern formation, cell division, and cell death in eye imaginal discs. Dev. Biol. 150: 381-396.

Binari, R. and N. Perrimon. 1994. Stripe-specific regulation of pair-rule genes by hopscotch, a putative Jak family tyrosine kinase in Drosophila. Genes \& Dev. 8: 300-312.

Bonni, A., Y. Sun, V.M. Nadal, A. Bhatt, D.A. Frank, I. Rozovsky, N. Stahl, G.D. Yancopoulos and M.E. Greenberg. 1997. Regulation of gliagenesis in the central nervous system by the JAK-STAT signaling pathway. Science 278: 477483.

Brand, A.H. and N. Perrimon. 1993. Targeted gene expression as a means of altering cell fates and generating dominant phenotypes. Development 118: 401-415.

Brodsky, M.H. and H. Steller. 1996. Positional information along the dorsal-ventral axis of the Drosophila eye: Graded expression of the four-jointed gene. Dev. Biol. 173: 428-446.

Chanut, F. and U. Heberlein. 1995. Role of the morphogenetic furrow in establishing polarity in the Drosophila eye. Development 121: 4085-4094.

Cho, K.-O. and K.-W. Choi. 1998. Fringe is essential for mirror symmetry and morphogenesis in the Drosophila eye. Nature 396: 272-276.

Choi, K.W., B. Mozer, and S. Benzer. 1996. Independent determination of symmetry and polarity in the Drosophila eye. Proc. Natl. Acad. Sci. 93: 5737-5741.

Cooper, M.T.D. and S.J. Bray. 1999. Frizzled regulation of Notch signaling polarizes cell fate in the Drosophila eye. Nature 397: 526-530.

Darnell, J.J. 1997. STATs and gene regulation. Science 277: 1630-1635.

Davis, I., C.H. Girdham, and P.H. O'Farrell. 1995. A nuclear GFP that marks nuclei in living Drosophila embryos: Maternal supply overcomes a delay in the appearance of zygotic 
fluorescence. Dev. Biol. 170: 726-729.

Domínguez, M. and J.F. de Celis. 1998. A dorsal/ventral boundary established by Notch controls growth and polarity in the Drosophila eye. Nature 396: 276-278.

Durbin, J.E., R. Hackenmiller, M.C. Simon, and D.E. Levy. 1996. Targeted disruption of the mouse Stat 1 gene results in compromised innate immunity to viral disease. Cell 84: 443-450.

Fanto, M. and M. Mlodzik. 1999. Asymmetric Notch activation specifies photoreceptors R 3 and R4 and planar polarity in the Drosophila eye. Nature 397: 523-526.

Golic, K.G. and S. Lindquist. 1989. The FLP recombinase of yeast catalyses site-specific recombination in the Drosophila genome. Cell 59: 499-509.

Hanratty, W.P. and C.R. Dearolf 1993. The Drosophila Tumorous-lethal hematopoietic oncogene is a dominant mutation in the hopscotch locus. Mol. \& Gen. Genet. 238: 33-37.

Harrison, D.A., R. Binari, T.S. Nahreini, M. Gilman, and N. Perrimon. 1995. Activation of a Drosophila Janus kinase (JAK) causes hematopoietic neoplasia and developmental defects. EMBO J. 14: 2857-2865.

Harrison, D.A., P.E. McCoon, R. Binari, M. Gilman, and N. Perrimon. 1998. Drosophila unpaired encodes a secreted protein that activates the JAK signaling pathway. Genes \& Dev. 12: 3252-3263.

Heberlein, U., E. Borod, and F. Chanut. 1998. Dorsoventral patterning in the Drosophila retina by wingless. Development 125: $567-577$.

Hou, X.S., M.B. Melnick, and N. Perrimon. 1996. Marelle acts downstream of the Drosophila HOP/JAK kinase and encodes a protein similar to the mammalian STATs. Cell 84: 411-419.

Ito, K., W. Awano, K. Suzuki, Y. Hiromi, and D. Yamamoto. 1997. The Drosophila mushroom body is a quadruple structure of clonal units each of which contains a virtually identical set of neurones and glial cells. Development 124: 761771.

Kaplan, M.H., Y.L. Sun, T. Hoey, and M.J. Grusby. 1996. Impaired IL-12 responses and enhanced development of Th2 cells in Stat4- deficient mice. Nature 382: 174-177.

Liu, X., G.W. Robinson, K.U. Wagner, L. Garrett, B.A. Wynshaw, and L. Hennighausen. 1997. Stat5a is mandatory for adult mammary gland development and lactogenesis. Genes \& Dev. 11: 179-186.

Luo, H., W.P. Hanratty, and C.R. Dearolf. 1995. An amino acid substitution in the Drosophila hopTum-1 Jak kinase causes leukemia-like hematopoietic defects. EMBO J. 14: 1412-1420.

$\mathrm{Ma}, \mathrm{C}$. and K. Moses. 1995. Wingless and patched are negative regulators of the morphogenetic furrow and can affect tissue polarity in the developing Drosophila compound eye. Development 121: 2279-2289.

Macchi, P., A. Villa, S. Gillani, M.G. Sacco, A. Frattini, F. Porta, A.G. Ugazio, J.A. Johnston, F. Candotti, J.J. O'Shea, P. Vezzoni, and L.D. Notarangelo. 1995. Mutations of Jak-3 gene in patients with autosomal severe combined immune deficiency (SCID). Nature 377: 65-68.

McNeill, H., C.H. Yang, M. Brodsky, J. Ungos, and M.A. Simon. 1997. mirror encodes a novel PBX-class homeoprotein that functions in the definition of the dorsal-ventral border in the Drosophila eye. Genes \& Dev. 11: 1073-1082.

O'Shea, J.J. 1997. Jaks, STATs, cytokine signal transduction, and immunoregulation: Are we there yet? Immunity 7: 111.

Papayannopoulos, V., A. Tomlinson, V.M. Panin, C. Rauskolb, and K.D. Irvine. 1998. Dorsal-ventral signaling in the Drosophila eye. Science 281: 2031-2034.
Perrimon, N. and A.P. Mahowald. 1986. 1(1)hopscotch, A larvalpupal zygotic lethal with a specific maternal effect on segmentation in Drosophila. Dev. Biol. 118: 28-41.

Reifegerste, R., C. Ma, and K. Moses. 1997. A polarity field is established early in the development of the Drosophila compound eye. Mech. Dev. 68: 69-79.

Robinow, S. and K. White. 1991. Characterization and spatial distribution of the ELAV protein during Drosophila melanogaster development. J. Neurobiol. 22: 443-461.

Schindler, C., K. Shuai, V.R. Prezioso, and J.E. Darnell. 1992. Interferon-dependent tyrosine phosphorylation of a latent cytoplasmic transcription factor. Science 257: 809-813.

Shimoda, K., J. van Dearsen, M.Y. Sangster, S.R. Sarawar, R.T. Carson, R.A. Tripp, C. Chu, F.W. Quelle, T. Nosaka, D.A. Vignali, P.C. Doherty, G. Grosveld, W.E. Paul, and J.N. Ihle. 1996. Lack of IL-4-induced Th2 response and IgE class switching in mice with disrupted Stat6 gene. Nature 380: 630-633.

Spana, E.P. and C.Q. Doe. 1995. The prospero transcription factor is asymmetrically localized to the cell cortex during neuroblast mitosis in Drosophila. Development 121: 3187-3195.

Struhl, G. and K. Basler. 1993. Organizing activity of wingless protein in Drosophila. Cell 72: 527-540.

Strutt, D.I. and M. Mlodzik. 1995. Ommatidial polarity in the Drosophila eye is determined by the direction of furrow progression and local interactions. Development 121: 4247-4256.

Sweitzer, S.M., S. Calvo, M.H. Kraus, D.S. Finbloom, and A.C. Larner 1995. Characterization of a Stat-like DNA binding activity in Drosophila melanogaster. J. Biol. Chem. 270: 16510-16513.

Tomlinson, A. and D.F. Ready. 1987. Neuronal differentiation in the Drosophila ommatidium. Dev. Biol. 120: 366-376.

Treisman, J.E. and G.M. Rubin. 1995. wingless inhibits morphogenetic furrow movement in the Drosophila eye disc. Development 121: 3519-3527.

Vinson, C.R., S. Conover, and P.N. Adler. 1989. A Drosophila tissue polarity locus encodes a protein containing seven potential transmembrane domains. Nature 338: 263-264.

Wehrli, M. and A. Tomlinson. 1998. Independent regulation of anterior/posterior and equatorial/polar polarity in the Drosophila eye; evidence for the involvement of Wnt signaling in the equatorial/polar axis. Development 125: 1421-1432.

Wolff, T. and D.F. Ready. 1993. Pattern formation in the Drosophila retina. In The development of Drosophila melanogaster (ed. M. Bate and A. Martinez-Arias). pp. 1277-1326, Cold Spring Harbor Laboratory Press, Cold Spring Harbor, NY.

Xu, T. and G.M. Rubin. 1993. Analysis of genetic mosaics in developing and adult Drosophila tissues. Development 117: $1223-1237$

Yan, R., H. Luo, J. Darnell, and C.R. Dearolf. 1996a. A JAKSTAT pathway regulates wing vein formation in Drosophila. Proc. Natl. Acad. Sci. 93: 5842-5847.

Yan, R., S. Small, C. Desplan, C.R. Dearolf, and J.J. Darnell. 1996b. Identification of a Stat gene that functions in Drosophila development. Cell 84: 421-430.

Zheng, L., J. Zhang, and R.W. Carthew. 1995. frizzled regulates mirror-symmetric pattern formation in the Drosophila eye. Development 121: 3045-3055. 


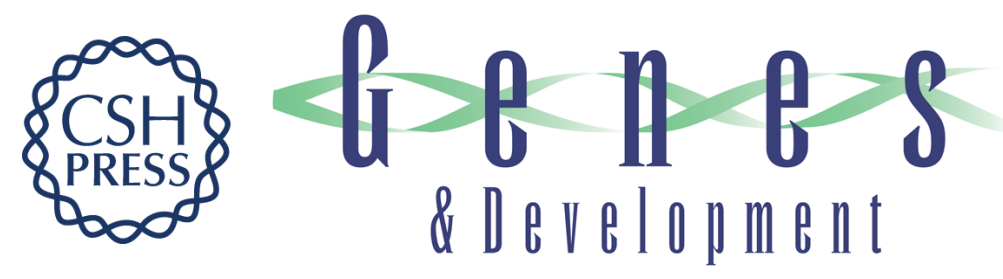

\section{Polarity determination in the Drosophila eye: a novel role for Unpaired and JAK/STAT signaling}

Martin P. Zeidler, Norbert Perrimon and David I. Strutt

Genes Dev. 1999, 13:

References This article cites 46 articles, 22 of which can be accessed free at:

http://genesdev.cshlp.org/content/13/10/1342.full.html\#ref-list-1

License

Email Alerting

Receive free email alerts when new articles cite this article - sign up in the box at the top

Service right corner of the article or click here.

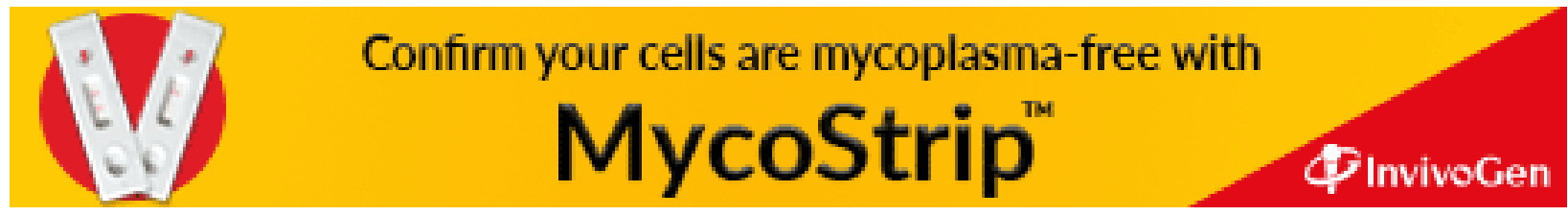

